\title{
Clinical AND EPIDEMIOLOGICAL ASPECTS OF PARVOVIRUS B19 INFECTIONS IN IRELAND, JANUARY 1996-JUNE 2008
}

\author{
N Nicolay (nathalie.nicolay@hse.ie) $)^{1,2}$, S Cotter $^{1}$ \\ 1. Health Protection Surveillance Centre (HPSC), Dublin, Ireland \\ 2. European Programme for Intervention Epidemiology Training (EPIET)
}

Parvovirus B19 infection may be mistakenly reported as measles or rubella if laboratory testing is not performed. As Europe is seeking to eliminate measles, an accurate diagnosis of fever/rash illnesses is needed. The main purpose of this study was to describe the epidemiological pattern of parvovirus B19, a common cause of rash, in Ireland between January 1996 and June 2008, using times series analysis of laboratory diagnostic data from the National Virus Reference Laboratory. Most diagnostic tests for presumptive parvovirus B19 infection were done in children under the age of five years and in women of child-bearing age (between 20-39 years-old). As a consequence, most of the acute diagnoses of B19 infection were made in these populations. The most commonly reported reasons for testing were: clinical presentation with rash, acute arthritis, influenza-like symptoms or pregnancy. The time series analysis identified seasonal trends in parvovirus B19 infection, with annual cycles peaking in late winter/spring and a six-year cycle for parvovirus B19 outbreaks in Ireland.

\section{Introduction}

Human parvovirus B19 infection is the cause of erythema infectiosum, or "slapped cheek" disease, a fever/rash illness occurring most frequently in childhood. The clinical presentation of parvovirus B19 infection is sometimes mistakenly diagnosed as rubella or measles. Although typically a mild, self-limiting disease, the infection can cause severe adverse outcomes in certain groups. In pregnant women infection can result in foetal death or hydrops foetalis, and among individuals with haematological disorders, complications such as anaemia or aplastic crisis can occur [1].

An accurate diagnosis of fever/rash illness is necessary not only for case management but also for public health control activities, particularly in outbreak situations in which measles or rubella is suspected [2]. As Europe seeks to eliminate measles as part of the World Health Organization's European strategy it is important that fever/rash illnesses are accurately diagnosed and that parvovirus B19 infection is not mistakenly reported as measles or rubella $[3,4]$. The lack of commercially available, convenient and noninvasive diagnostic tests for parvovirus B19 may play a role in the misdiagnosis of measles and rubella cases $[5,6]$. Because many individuals with fever/rash illnesses are not routinely tested, each year many notified measles and rubella cases are not laboratoryconfirmed. In Ireland in 2007, for instance, only 20 of 53 notified measles cases were laboratory-confirmed [7].
No data are available on the prevalence of parvovirus B19 infection in the Irish population, nor on the pattern of disease incidence in Ireland. As the infection is often asymptomatic, it is difficult to have a comprehensive picture of disease incidence. Due to the limited information available to us on the epidemiology of B19 in Ireland, we collaborated with the National Virus Reference Laboratory (NVRL) on a study to describe which population groups were most commonly tested for parvovirus B19, and to describe, using the pattern of laboratory diagnosis of acute infection, the epidemic pattern of acute parvovirus B19 in Ireland between January 1996 and June 2008.

\section{Materials and methods}

The NVRL is the main diagnostic facility in Ireland for the diagnosis of parvovirus B19 infection. During our study we identified three regional hospitals which also offer local testing but they represented a minority of all tests done in Ireland. Upon suspicion of acute parvovirus B19 disease, a clinician may request diagnostic testing. Serum samples are sent to the diagnostic laboratory either directly, by individual clinicians, or via any of the hospitals' microbiological laboratories. Acute infection is diagnosed by the detection of parvovirus B19-specific immunoglobulin $M$ (IgM). These samples are tested by enzyme immunoassay (EIA) in serum or plasma (Parvovirus B19 IgM (mu capture) EIA, Biotrin International).

To estimate the incidence of laboratory-confirmed disease in Ireland, information relating to each individual testing positive for parvovirus B19-specific IgM was extracted from the laboratory information system at the NVRL and sent to the Health Protection Surveillance Centre (HPSC) for analysis. The initial database consisted of a listing of all the positive tests performed at the NVRL with details on place and date of blood samples, age and sex of the patient, clinical symptoms associated with disease or an underlying condition consistent with this diagnosis, results for parvovirus IgM testing and parvovirus IgG testing in laboratory-confirmed acute cases. To eliminate duplicate results originating from patients presenting to clinicians for the same event, all the line listings were reviewed. Duplicates were defined as similar records based on same dates of birth, sex, place of testing, within a period of three days. Duplicate records were excluded from subsequent analysis. Age and sex distribution, clinical features according to the age of the patient, time and place of occurrence of positive tests for parvovirus IgM were described. 
Times series analysis was carried out for the series of laboratoryconfirmed acute parvovirus B19 cases reported per month. A linear function was used for analysing secular trends. In order to describe cycles and seasonality in the series, secular trend was removed and data were log transformed for stabilising minor changes in the variance along the series. From the new working series, cyclic components were identified using a Fast Fourier Transformation. Cycles with energy above the upper limit of the $95 \%$ confidence interval for the mean energy of the cycles were estimated using the least squares period. An equation with the following structure was obtained:

$$
y=f(x)+\sum A \cos \left[2 \pi\left(\frac{x-\theta}{p}\right)\right]
$$

where $A$ is the amplitude, $\theta$ is the phase and $p$ is the period of cosine function of significant cycles.

\section{F I G U R E 1}

Requests for parvovirus B19-specific immunoglobulin $\mathrm{M}$ detection by year, National Virus Reference Laboratory, Ireland, January 1996-June $2008(\mathrm{n}=11,437)$



\section{F I G U R E 2}

Requests for parvovirus B19-specific immunoglobulin $M$ detection by age group and sex, National Virus Reference Laboratory, Ireland, January 1996 -June $2008(n=11,437)$

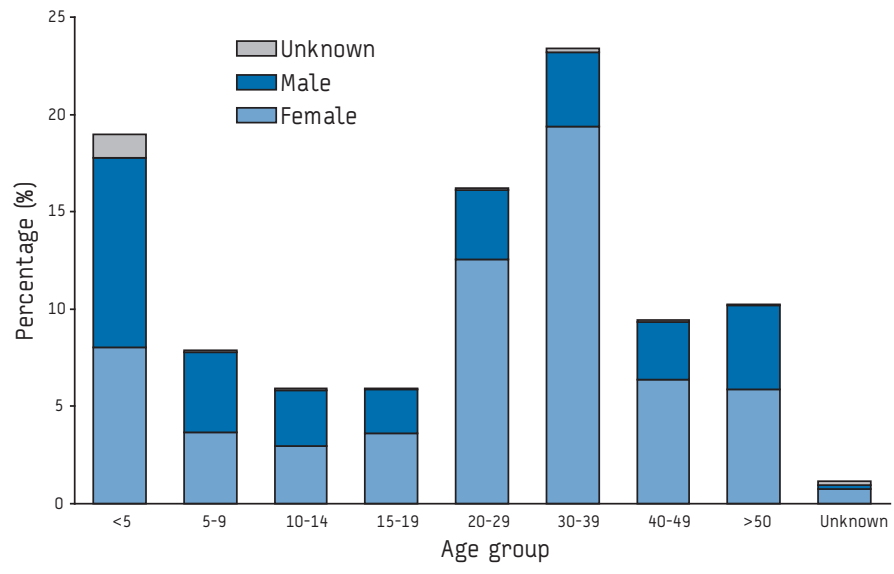

Analyses were performed using Stata V9.2 (Stata Corporation) and the Fourier Transformation was done using R V2.8.1 ( $R$ foundation, www.r-project.org).

\section{Results}

\section{Descriptive results}

Parvovirus B19 tests undertaken by NVRL

Between January 1996 and June 2008, a total of 12,430 tests for parvovirus B19 were carried out at the NVRL. Of those, 546

F I G U R E 3

Number of positive tests for parvovirus B19-specific

Immunoglobulin $M$ by age and sex, National Virus Reference Laboratory, Ireland, January 1996-June $2008(n=497)^{*}$

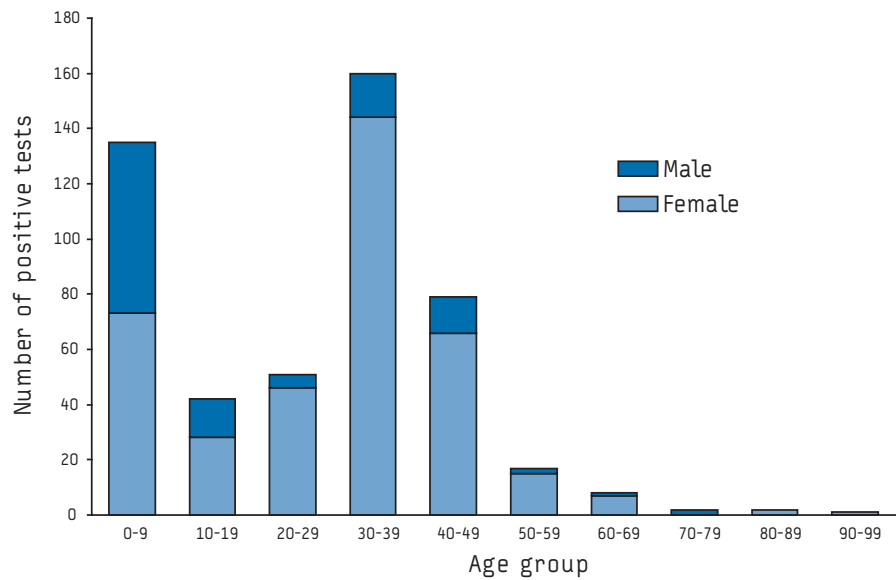

* Age is unknown for 15 patients and sex is unknown for two patients under the age of 10 years

T A B L E

Most common clinical associations observed in patients with parvovirus B19-specific immmunoglobulin $M$ by age group, National Virus Reference Laboratory, Ireland, January 1996-June 2008

\begin{tabular}{|c|c|c|c|}
\hline Age group & $<\begin{array}{c}15 \text { years } \\
(n=60)^{*}\end{array}$ & $\begin{array}{c}>=15 \text { years } \\
(n=190)^{*}\end{array}$ & $\begin{array}{c}\text { Total } \\
(n=198)^{*}\end{array}$ \\
\hline Clinical features & \multicolumn{3}{|c|}{ No. of reports (\% Total) } \\
\hline Rash & $28(34)$ & $52(63)$ & $83(42)$ \\
\hline “Slapped cheek” appearance & $5(71)$ & $2(28)$ & $7(3.5)$ \\
\hline Acute arthritis & 7 (16) & $35(79)$ & $44(22)$ \\
\hline Fever & $9(32)$ & $18(64)$ & $28(14)$ \\
\hline Influenza-like symptoms & $1(4)$ & $24(92)$ & $26(13)$ \\
\hline Pregnancy & 0 & $23(96)$ & $24(12)$ \\
\hline Intrauterine death & 0 & $8(100)$ & $8(4)$ \\
\hline Hydrops fetalis & 0 & $1(100)$ & $1(0.5)$ \\
\hline Anaemia & $11(73)$ & $4(26)$ & $15(8)$ \\
\hline Haemophilia/Sickle cell anaemia & $7(70)$ & $1(10)$ & $10(5)$ \\
\hline Sore throat & $3(42)$ & $4(57)$ & $7(4)$ \\
\hline Lymphadenopathy & 0 & $5(100)$ & $5(3)$ \\
\hline Headache & $3(75)$ & $1(25)$ & $4(2)$ \\
\hline Bone marrow transplant & $2(100)$ & 0 & $2(1)$ \\
\hline
\end{tabular}

* The total number of symptoms exceeds the total number of cases as more than one symptom could be mentioned per case.

$\dagger$ Age is unknown for eight patients for whom clinical details were given. 
were positive, and 993 duplicates tests were identified. Following de-duplication, 514 (4.5\%) acute cases of parvovirus B19 were identified out of 11,437 tests performed (Figure 1 ).

The number of test requests increased over the time period, from 500 requests in January 1996 to 1,388 in 2007 . The proportion of IgM-positive tests varied depending on the year and the seasonality of parvovirus B19 in the community.

Most samples (27\%) originated from children under the age of 10 years, the majority of whom were under five years old $(19 \%$ of all events). The next largest age group was the age group of 3039 year-olds (23\%), followed by $20-29$ year-olds (16\%). Females were more likely to be tested than males ( $64 \%$ of all requests), most marked in the women of child-bearing age; $77 \%$ of requests were made for the 30-39 year-old group and $83 \%$ for the $20-29$ year-olds (Figure 2).

\section{Positive tests $(n=514)$}

Overall, $76 \%$ of all positive tests occurred in female patients, giving a female:male ratio of 3.3:1 (Figure 3). The median age of all cases for whom the age was known was 31 years (range 7 days to 92 years); information on age was missing for 15 positive patients. Males tested positive were more likely to be younger than females $(p<10-3)$. The median age for male patients was nine years and for female patients 33 years.

A total of 168 positive tests (32.7\%) belonged to patients under the age of 15 years; 137 positive tests (21.3\%) occurred in children between 0 and 9 years of age, with equal distribution between male and female children; 160 (31.1\%) positive tests originated from patients between 30 and 39 years of age, $90 \%$ of whom were female. Of the 514 IgM-positive cases, 300 (58.4\%) were also positive for IgG.

\section{Regional distribution of events}

There was marked regional distribution of positive tests. Most of the positive IgM samples (60.3\%) were from the former Eastern Health Board region (encompassing the Dublin metropolitan region among others). Samples originating from the North Western Health Board, the Midland Health Board and the North Eastern Health Board represented $7.8 \%, 5.8 \%$ and $5.4 \%$ of positive tests, respectively. The lowest number of positive test results came from samples taken in the Western Health Board and in the Mid-Western Health Board regions (2.4\%).

Clinical information provided with diagnostic samples

Clinical information accompanied the request for parvovirus B19-specific IgM testing for 198 (38.5\%) of patients who tested positive for parvovirus IgM (Table). Parvovirus B19 infection was characterised by a variable combination of symptoms: rash, influenza-like symptoms, joint pain and haematologic abnormalities were often reported.

The most common symptom reported in parvovirus B19 IgMpositive patients was a rash $(n=83,42 \%)$ but the typical "slapped cheek" appearance was mentioned in only seven patients as shown in the Table. Joint pain was reported in 44 patients $(22.1 \%)$ and was more common in adults than children. Fever was the third most commonly reported symptom (14\%). Anaemia was the reason for testing for 15 patients (8\%), and these were mainly young patients with $11(68 \%)$ under the age of 15 years. Among these 11 , six had sickle cell anaemia and four had haemophilia.

Finally, 24 women (12\%) were tested because they were pregnant (the specific circumstances however were not reported for the majority of them). Among those women, eight experienced an abortion or a miscarriage (one in 1996, two in 1999, one in 2000 , one in 2004, two in 2005 and one in 2008).

Other documented symptoms reported at the time of diagnostic test request were varied and often unspecific, including reporting influenza-like symptoms, fever and fatigue (30 patients, 15.1\%). Three parvovirus infections occurred in patients known to be immunocompromised (Hodgkin's disease, renal transplant). One case was due to occupational exposure (unspecified).

\section{F I G U R E 4}

Monthly series of positive parvovirus B19-specific immunoglobulin M, National Reference Virus Laboratory, Ireland, January 1996-June 2008

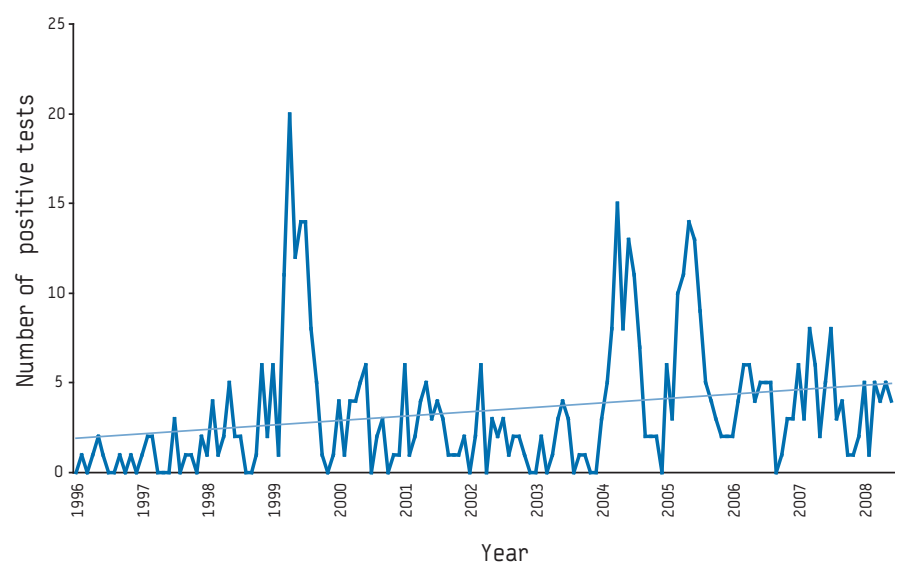

F I G U R E 5

Periodogram of parvovirus B19 monthly series, Ireland, January 1996-June 2008*

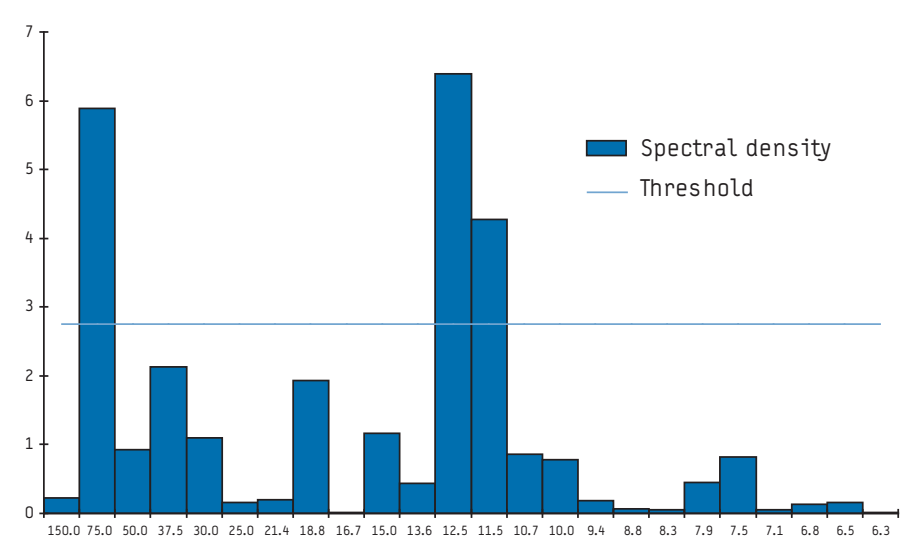

* Periods were identified using Fast Fourier Transformation. 


\section{Seasonal pattern and periodicity of epidemic years}

The 514 acute cases of parvovirus B19 were reported over a period of 149 months between January 1996 to June 2008 (Figure 4). The average number of cases diagnosed per month was 3.4 (standard deviation (SD) +/- 3.7). The maximum number of cases was diagnosed during April $1999(n=20)$. An increasing trend in the number of acute cases was observed over the study period.

The Fast Fourier Transformation analysis identified two statistically significant components, one annual and the other every 75 months, i.e. approximately six years (Figure 5). Annual cycles peak during winter/spring : each year the majority of cases occurred between March and July.

\section{Discussion}

In Ireland as well as in most other European countries, parvovirus B19 infection is not a notifiable disease, and neither clinical descriptive data nor epidemiological data are readily available. To our knowledge, this is the first Irish study which attempts to describe the clinical and epidemiological pattern of parvovirus infection based on acute cases identified by the reference laboratory.

Our initial objective was to describe the epidemic pattern of parvovirus infection. We identified a periodicity of six years and an annual seasonality pattern, with most cases diagnosed between March and July. Our results showed that there was no sex difference in testing patterns in young children, which was to be expected as parvovirus B19 affects both sexes equally. In older age groups, testing is done predominantly in women; during pregnancy, the disease can lead to severe adverse outcomes for the foetus. Of 24 parvovirus B19 positive women known to be pregnant in this report, nine experienced a foetal loss, of whom four had acquired the infection during a time of increased incidence (two in 1999 and two in 2005). A prospective study in the United Kingdom estimated the risk of transplacental infection at 30\%, with $5-9 \%$ of foetal loss reported [8]. As the number of pregnancies as well as the coverage of the screening in pregnant women are unknown for our study period, the data are not comparable. Nevertheless, they remind us of how severe the disease can be in pregnant women and of the consequences for the foetus. A better knowledge of the prevalence of parvovirus B19 in the Irish population is needed for further interpretation.

Our data were obtained from the Irish National Reference Virus Laboratory. It is situated in Dublin, the metropolitan area of which accounts for approximately one quarter of the Irish population. Although this may explain in part why the majority of tests came from the Eastern region (including Dublin), it does not fully explain the under-representation of other regions in these results. The extent to which other alternative testing may be done at local level is a possibility. However, we could identify only three other regional hospitals that undertook testing, and the number of positive tests performed there during the period under investigation was small $(n=50)$. Despite this lack of regional representation we believe that our data represent a fair approximation of the current endemic situation of symptomatic parvovirus B19 in Ireland. The overrepresentation of the Dublin area most likely reflects increased awareness of testing and submission to the laboratory by both clinicians and the local hospital laboratories. There is no reason to suspect that there is a connection between geography and susceptibility to this common illness. The epidemiology of parvovirus B19 infection has many of the characteristics of other common childhood communicable diseases which were common in the pre-vaccine aera (e.g. measles, rubella or mumps), all of which demonstrated outbreak years followed by periods of low incidence before the next outbreak. However, our data are unlikely to be fully representative of the true distribution of acute parvovirus B19 infection in the general Irish population due to a testing bias for certain population groups (young children and women attending maternity hospitals). It is likely that people tested in the present study represent the most seriously affected cases or the population considered to be most at risk.

Because human parvovirus infection is not a notifiable disease in Ireland, we cannot test the assumption that IgM-positive results from a reference laboratory are representative of the pattern of acute infection in the community. Nevertheless previous studies have used data from reference laboratories to describe the seasonality of parvovirus B19 $[9,10]$. In Ireland, measles is a notifiable disease. By comparing the pattern of measles-specific positive samples tested by NVRL with the distribution pattern of measles notifications to the HPSC between 2000 and 2008, we find a similar trend, with an increase in laboratory testing for measles during periods of increased notification, thus supporting the main assumption we made for parvovirus B19. Published data on the seasonal activity of parvovirus B19 in temperate countries are limited. According to our data most of the cases occurred during winter and late spring. Based on the times series analysis, the periodicity of epidemic years is six years, which is concordant with data published for some other developed countries [10-12]. However we cannot exclude that the pattern of the disease may present with one or two consecutive epidemic years: both 2004 and 2005 had a substantial number of positive tests. Even though the data from a reference laboratory are not as informative as the data which could be provided by a national surveillance system, they can provide helpful insight into the epidemiological pattern of non-notifiable diseases. Awareness of the normal epidemiology of parvovirus B19 can help clinicians who are confronted with patients with rash illnesses in the differential diagnosis for all compatible rash illness, especially measles.

Assuming a periodicity of six years, we can expect the next epidemic year in the coming two years (2011). We hope that this study will alert clinicians and increase diagnostic testing of all rash illnesses as they present. Numerous studies have highlighted the difficulty in making an accurate diagnosis of rash diseases $[5,6,13,14]$. The development of new and non-invasive technology that allows the sampling of oral fluid to diagnose viral infections such as measles [15], mumps [16], and hepatitis A and B [17] has increased the number of laboratory-confirmed diagnoses in many countries. Such testing has been found to be both sensitive and specific and is routinely used in many countries in case diagnosis. Commercial tests for the serological diagnosis of parvovirus B19 are available, but none are validated for use on oral fluid samples. Such a diagnostic tool would be invaluable, particularly when investigating fever/rash illnesses in young children [2] who are not so ill as to require hospitalisation but are usually seen by general practitioners (GPs) in the community. Anecdotal reports indicate that Irish GPs are reluctant to undertake phlebotomy in such paediatric cases and hence accurate laboratory confirmation of fever illness is often not done. A recently developed test to diagnose acute parvovirus B19 infection using oral fluid samples is now being assessed by the NVRL as part of a collaborative study with HPSC and clinicians around the country. 
The value in adding parvovirus testing to enhanced measles surveillance has been demonstrated in South Australia where measles, rubella and parvovirus testing are included in routine measles surveillance. Despite a low overall rate of measles testing, this was particularly obvious in an inter-epidemic period when most notified measles cases were not measles [18]. Between 2\% and $10 \%$ of suspected measles cases tested in South Australia between 1999 and 2004, were parvovirus B19 cases [19]. An added value was also shown when including parvovirus testing in the rubella surveillance programme [14].

In countries in the elimination phase for measles and rubella, a better knowledge of the epidemiology of parvovirus B19 may help clinicians in the differential diagnosis of common rash diseases. Meanwhile, a better laboratory confirmation of common rash illnesses is required to improve the quality of national data and public health action. The anticipated availability of an oral fluid test for parvovirus B19 will be useful in this aim.

Vivamus tempor mi quis quam. Fusce tempus, ante sed tincidunt ornare, nisi urna viverra enim, eget venenatis dui ante ut eros.

\section{Acknowledgements}

We would like to acknowledge Dr Jeff Connell and Joanne Moran, National Virus Reference Laboratory, Dublin, Ireland for providing the data as well as Dr Francisco Luquero for his statistical support.

\section{References}

1. Young NS, Brown KE. Parvovirus B19. N Engl J Med. 2004;350(6):586-97.

2. Goncalves G, Correia AM, Palminha P, Rebelo-Andrade H, Alves A. Outbreaks caused by parvovirus B19 in three Portuguese schools. Euro Surveill. 2005;10(6):pii=549. Available from: http://www.eurosurveillance.org/ ViewArticle.aspx?ArticleId $=549$

3. Strategic plan for measles and congenital rubella infection in the WHO European Region. Copenhagen: World Health organisation Regional Office for Europe; 2003. Available from: http://www.euro.who.int/document/e81567.pdf

4. Surveillance guidelines for measles and congenital rubella infection in the WHO European Region. Copenhagen: World Health Organization Regional Office for Europe; 2003. Available from: http://www.euro.who.int/document/e82183. pdf

5. Davidkin I, Valle M, Peltola H, Hovi T, Paunio M, Roivainen M, et al. Etiology of measles- and rubella-like illnesses in measles, mumps, and rubellavaccinated children. J Infect Dis. 1998;178(6):1567-70.

6. Ramsay M, Reacher M, O'Flynn C, Buttery R, Hadden F, Cohen B, et al. Causes of morbilliform rash in a highly immunised English population. Arch Dis Child. 2002;87(3):202-6.

7. Health Protection Surveillance Centre. Annual Report 2007. Available from: http://www.ndsc.ie/hpsc/AboutHPSC/AnnualReports/File,3377,en.pdf.

8. Miller E, Fairley CK, Cohen BJ, Seng C. Immediate and long term outcome of human parvovirus B19 infection in pregnancy. Br J Obstet Gynaecol. 1998;105(2):174-8.

9. Enders M, Weidner A, Enders G. Current epidemiological aspects of human parvovirus B19 infection during pregnancy and childhood in the western part of Germany. Epidemiol Infect. 2007;135(4):563-9.

10. Kelly HA, Siebert D, Hammond R, Leydon J, Kiely P, Maskill W. The age-specific prevalence of human parvovirus immunity in Victoria, Australia compared with other parts of the world. Epidemiol Infect. 2000;124(3):449-57.

11. Vyse AJ, Andrews NJ, Hesketh LM, Pebody R. The burden of parvovirus B19 infection in women of childbearing age in England and Wales. Epidemiol Infect. 2007;135(8):1354-62.

12. Zaaijer HL, Koppelman MH, Farrington CP. Parvovirus B19 viraemia in Dutch blood donors. Epidemiol Infect. 2004;132(6):1161-6.

13. Davidkin I, Jokinen S, Paananen A, Leinikki P, Peltola H. Etiology of mumps-like illnesses in children and adolescents vaccinated for measles, mumps, and rubella. J Infect Dis. 2005;191(5):719-23.

14. Oliveira MI, Curti SP, Figueiredo CA, Afonso AM, Theobaldo M, Azevedo RS, et al. Rash after measles vaccination: laboratory analysis of cases reported in Sao Paulo, Brazil. Rev Saude Publica. 2002;36(2):155-9.
15. Perry KR, Brown DW, Parry JV, Panday S, Pipkin C, Richards A. Detection of measles, mumps, and rubella antibodies in saliva using antibody capture radioimmunoassay. J Med Virol. 1993;40(3):235-40.

16. Reid F, Hassan J, Irwin F, Waters A, Hall W, Connell J. Epidemiologic and diagnostic evaluation of a recent mumps outbreak using oral fluid samples. J Clin Virol. 2008;41(2):134-7.

17. Thieme T, Yoshihara P, Piacentini S, Beller M. Clinical evaluation of oral fluid samples for diagnosis of viral hepatitis. J Clin Microbiol. 1992;30(5):1076-9.

18. Lambert SB, Kelly HA, Andrews RM, Catton MC, Lynch PA, Leydon JA, et al Enhanced measles surveillance during an interepidemic period in Victoria. Med J Aust. 2000;172(3):114-8.

19. Kelly H, Leydon J. Letter to the editor: outbreaks caused by parvovirus B19. Euro Surveill. 2005;10(9): pii=565. Available from: http://www.eurosurveillance. org/ViewArticle.aspx?ArticleId $=565$

This article was published on 25 June 2009.

Citation style for this article: Nicolay N, Cotter S. Clinical and epidemiological aspects of parvovirus B19 infections in Ireland, January 1996-June 2008. Euro Surveill. 2009;14(25):pii=19249. Available online: http://www.eurosurveillance.org/ViewArticle. aspx?ArticleId $=19249$ 